Methods: The performance of Rheumatic? was tested using data from 175 patients from three university rheumatology centers covering two different settings: A.Risk-RA phase setting. Here, we tested whether Rheumatic? could predict the development of arthritis in 50 at risk-individuals with musculoskeletal complaints and anti-citrullinated protein antibody positivity from the KI (Karolinska Institutet) B.Early arthritis setting. Here, we tested whether Rheumatic? could predict the development of an immune-mediated rheumatic disease in i) EUMC (Erlangen) $\mathrm{n}=52$ patients and $\mathrm{ii}$ ) LUMC (Leiden) $\mathrm{n}=73$ patients.

In each setting, we examined the discriminative power of the total score with the Wilcoxon rank test and the area-under-the-receiver-operating-characteristic curve (AUC-ROC).

Results: In setting A, the total test score clearly differentiated between individuals developing arthritis or not, median 245 versus 163, $P<0.0001$, AUC-ROC = 75.3 (Figure 1). Also within patients with arthritis the Rheumatic? total score was significantly higher in patients developing an immune-mediated arthritic disease versus those who did not: median score EUMC 191 versus 107, $P<0.0001$, AUC$\mathrm{ROC}=79.0$, and LUMC 262 versus 212, $P<0.0001, \mathrm{AUC}-\mathrm{ROC}=53.6$
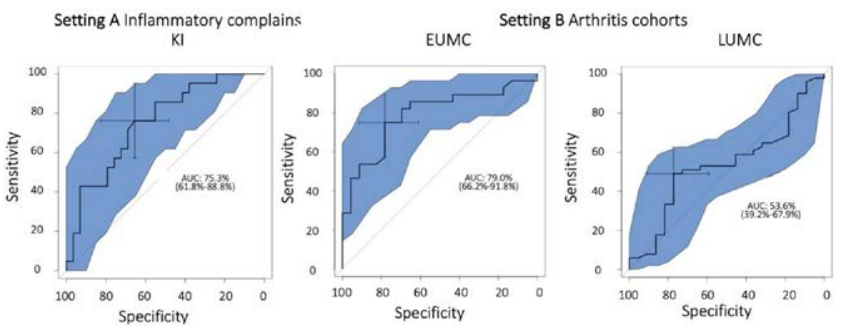

Figure 1. (Area under) the receiver operating curve for the total Rheumatic? score

Conclusion: Rheumatic? is a web-based patient-centered multilingual diagnostic tool capable of differentiating immune-mediated rheumatic conditions from other musculoskeletal problems. A following subject of research is how the tool performs in a population-wide setting.

REFERENCES:

[1] Knitza J. et al. Mobile Health in Rheumatology: A Patient Survey Study Exploring Usage, Preferences, Barriers and eHealth Literacy. JMIR mHealth and uHealth. 2020.

[2] https://rheumatic.elsa.science/en/

Acknowledgements: This project has received funding from EIT Health. EIT Health is supported by the European Institute of Innovation and Technology (EIT), a body of the European Union that receives support from the European Union's Horizon 2020 Research and Innovation program.

This project has received funding from the Innovative Medicines Initiative 2 Joint Undertaking under grant agreement No 777357, RTCure.

Disclosure of Interests: Rachel Knevel: None declared, Johannes Knitza: None declared, Aase Hensvold: None declared, Alexandra Circiumaru: None declared, Tor Bruce Employee of: Ocean Observations, Sebastian Evans Employee of: Elsa Science, Tjardo Maarseveen: None declared, Marc Maurits: None declared, Liesbeth Beaart- van de Voorde: None declared, David Simon: None declared, Arnd Kleyer: None declared, Martina Johannesson: None declared, Georg Schett: None declared, Thomas Huizinga: None declared, Sofia Svanteson Employee of: Elsa Science, Alexandra Lindfors Employee of: Ocean Observations, Lars Klareskog: None declared, Anca Catrina: None declared DOI: 10.1136/annrheumdis-2021-eular.1118

\section{OP0148 $\quad$ SPATIOTEMPORAL DYNAMICS OF BONE LOSS BEFORE AND AFTER THE ONSET OF RHEUMATOID ARTHRITIS}

K. Tascilar ${ }^{1}$, D. Simon ${ }^{1}$, A. M. Liphardt ${ }^{1}$, T. Meinderink ${ }^{1}$, S. Bayat ${ }^{1}$, J. Rech $^{1}$, A. Hueber ${ }^{2}$, G. Krönke ${ }^{1}$, G. Schett ${ }^{1}$, A. Kleyer ${ }^{1} .{ }^{1}$ Friedrich-Alexander; University (FAU) Erlangen-Nürnberg and Universitätsklinikum Erlangen, 91054 Erlangen, Department of Internal Medicine 3 - Rheumatology and Immunology, Erlangen, Germany; ${ }^{2}$ Solzialstiftung Bamberg, Sektion Rheumatologie, Bamberg, Germany

Background: Rheumatoid Arthritis (RA) is preceded by a clinically silent prephase characterized by autoimmunity against anti-modified protein antibodies including anti-citrullinated protein antibodies (ACPA). At this pre-stage patients already experience significant loss of volumetric peripheral bone mineral density (vBMD) compared to healthy controls measured by high-resolution peripheral quantitative computed tomography (HR-pQCT) (1-2). However, the longitudinal course of vBMD changes during the preclinical phase, after diagnosis, and its association with time to disease onset have not been investigated.
Objectives: To longitudinally characterize the changes of metacarpal and radial VBMD before and after the clinical onset of RA and its association with time to onset of arthritis.

Methods: To explore the development of arthritis, we initiated a RA-at-risk cohort in 2011. (Ethics 334_16B). This prospective cohort includes adults positive for $\mathrm{CCP}-\mathrm{AB}$ with or without musculoskeletal symptoms, excluding arthritis. Participants are regularly followed with clinical examination and HR-pQCT imaging of the MCP and radial bone to monitor early bone changes. HR-pQCT images with low motion grade artefacts were analyzed to obtain the total (D100), cortica (DComp) and trabecular (DTrab) vBMD (D100) in $\mathrm{mg} \mathrm{HA} \mathrm{cm}^{3}$.

We descriptively analyzed the VBMD time course in patients who developed RA by fitting regression curves separately for the pre-clinical and clinical periods and estimated time-conditional marginal mean VBMDs for the 5-year peri-RA period. We analyzed time to diagnosis of clinical RA defined by the 2010 ACR/EULAR classification criteria using Cox regression models. Hazard ratios indicate the relative risk of clinical disease onset associated with 1 standard deviation reduction in bone density.

Results: 130 subjects (mean [SD] age 47.0 [12.2], 89 female [68\%]) between 2011 and 2020 were analyzed. Median (IQR) follow-up duration for the cohort was 18.6 (4.6-47.6) months. Participants underwent 233 HR-pQCT scans and 58 $(45 \%)$ underwent 2 to 6 scans with a median interval of $16.2(12.2-21.2)$ months $49(38 \%)$ patients who developed RA had a pre-diagnosis follow-up of 4.1 (2.513.4) months and post-diagnosis follow-up of 22.0 (8.8-38.9) months. The time course of scaled bone mineral densities depicted in Figure $1 \mathrm{~A}$ suggest that bone

Table 1. Relative risk of RA development in the total cohort; crude and age/sex adjusted hazard ratios for one standard-deviation reduction in vBMD.

\begin{tabular}{lcccc}
\hline \multicolumn{2}{c}{ Crude } & & Adjusted \\
\hline & HR $(95 \% \mathrm{Cl})$ & $\mathrm{P}$ & HR $(95 \% \mathrm{Cl})$ & $\mathrm{P}$ \\
\hline MCP.D-Comp & $1.16(0.86$ to 1.57$)$ & 0.336 & $1.20(0.89$ to 1.63$)$ & 0.229 \\
MCP.D-Trab & $1.14(0.83$ to 1.57$)$ & 0.405 & $1.17(0.85$ to 1.62$)$ & 0.341 \\
MCP.D100 & $1.16(0.83$ to 1.61$)$ & 0.392 & $1.21(0.86$ to 1.71$)$ & 0.265 \\
Rad.D-Comp & $1.42(0.97$ to 2.07$)$ & 0.071 & $1.66(1.07$ to 2.55$)$ & 0.023 \\
Rad.D-Trab & $1.20(0.87$ to 1.66$)$ & 0.257 & $1.23(0.88$ to 1.71$)$ & 0.223 \\
Rad.D100 & $1.43(0.99$ to 2.06$)$ & 0.056 & $1.52(1.03$ to 2.25$)$ & 0.033 \\
\hline
\end{tabular}

\section{A}

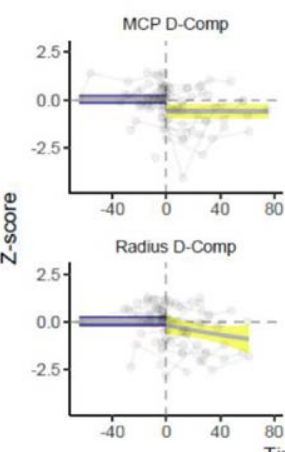

- Pre-RA - RA
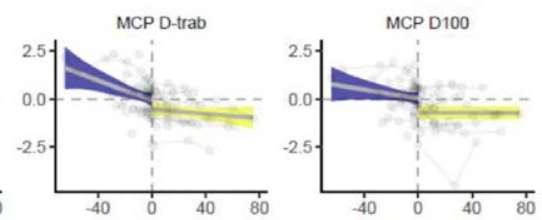

B
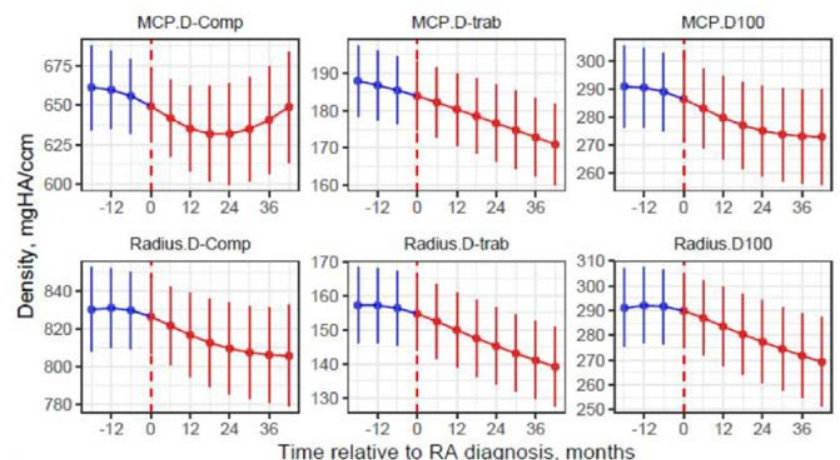

me relative to RA diagnosis, months

ingure-1 1 Tme Course ofvemD in the sub-cohort of patients who developed RA defined by the 2010 ACR/EULAR criteria. (A) Centered and scaled (z-score) peripheral vBMD values observed over time.
(B) Time-conditional marginal mean vBMD values estimated using mixed models. Time zero indicates the date of clinical RA diagnosis. 
density around the MCP joints deteriorate in the preclinical phase of RA, which is mostly prominent in the trabecular bone. Modelling (Figure 1B) suggests that trabecular bone loss around the MCP joints has a constant pace regardless of the clinical status. Whereas the radial bone densities are relatively stable in the preclinical phase and show a reduction after the clinical onset of RA. Age and sex adjusted hazard ratios $(95 \% \mathrm{Cl})$ for the risk of RA clinical onset were 1.52 (1.03 to $2.25)$ for radius D100 and 1.66 (1.07 to 2.55) for radius DComp (Table-1). Conclusion: Metacarpal bone showed a constant decline that started already in the pre-phase of RA and continued after its clinical onset. In contrast, bone loss in the radius was not observed in the pre-phase but started at onset of RA. Low radial VBMD in the pre-clinical phase, however, was associated with a higher risk of RA onset. These findings suggest different spatiotemporal dynamics of bone loss before and after RA onset

REFERENCES:

[1] Kleyer A. et. al. Ann Rheum Dis. 2014, 73:854-60

[2] Simon D. et. al. Ann Rheum Dis. 2020, doi:10.1002/art.41229

Disclosure of Interests: None declared

DOI: 10.1136/annrheumdis-2021-eular.4176

\section{OP0149 RELIABILITY AND RESPONSIVENESS OF TWO OMERACT WHOLE-BODY MRI SCORES OF ENTHESEAL AND JOINT INFLAMMATION IN THE KNEE REGION IN SPONDYLOARTHRITIS}

M. Wetterslev ${ }^{1,2}$, W. P. Maksymowych ${ }^{3,4}$, R. G. Lambert ${ }^{5,6}$, I. Eshed ${ }^{7}$, S. Juhl Pedersen $^{1}$, M. Stoenoiu ${ }^{8}$, S. Krabbe ${ }^{1}$, P. Bird ${ }^{9}$, V. Foltz ${ }^{10}$, A. J. Mathew ${ }^{1,2}$ F. Gandjbakhch ${ }^{10}$, J. Paschke ${ }^{4}$, P. Carron ${ }^{11,12}$, G. De Marco ${ }^{13,14}$, H. MarzoOrtega $^{13,14}$, A. E. F. Poulsen ${ }^{1}$, J. L. Jaremko ${ }^{5}$, P. G. Conaghan ${ }^{13,14}$, M. Østergaard ${ }^{1,2}$ on behalf of the OMERACT MRI in Arthritis Working Group. ${ }^{1}$ Rigshospitalet, Copenhagen Center for Arthritis Research, Center for Rheumatology and Spine Diseases, Copenhagen, Denmark; ${ }^{2}$ University of Copenhagen, Department of Clinical Medicine, Copenhagen, Denmark; ${ }^{3}$ University of Alberta, Department of Medicine, Edmonton, Alberta, Canada: ${ }^{4}$ CARE Arthritis, Edmonton, Alberta, Canada; ${ }^{5}$ University of Alberta, Department of Radiology and Diagnostic Imaging, Edmonton, Alberta Canada; ${ }^{6}$ Medical Imaging Consultants, Edmonton, Alberta, Canada; ${ }^{7} \mathrm{Tel}$ Aviv University, Department of Diagnostic Imaging, Sheba Medical Center, Affiliated to the Sackler School of Medicine, Israel; ${ }^{8}$ Université catholique de Louvain, Department of Rheumatology, Cliniques Universitaires SaintLuc, Institut de Recherche Expérimentale et Clinique, Brussels, Belgium; ${ }^{9}$ University of New South Wales, Division of Medicine, Sydney, Australia;

${ }^{10}$ Sorbonne University, Department of Rheumatology, APHP, PitiéSalpêtrière Hospital, Paris, France: ${ }^{11}$ Ghent University Hospital, Department of Rheumatology, Ghent, Belgium; ${ }^{12}$ Ghent University, VIB Inflammation Research Centre, Ghent, Belgium; ${ }^{13}$ University of Leeds, Leeds Institute of Rheumatic and Musculoskeletal medicine, Leeds, United Kingdom; ${ }^{14} \mathrm{NIHR}$ Leeds Biomedical Research Centre, Leeds Teaching Hospitals NHS Trust, Leeds, United Kingdom

Background: Inflammation in peripheral joints and entheses is common in spondyloarthritis $(\mathrm{SpA})$. Whole-body magnetic resonance imaging (WB-MRI) allows assessment of the overall inflammatory status of arthritis patients including joints and entheses. The OMERACT MRI Whole-body scoring system for Inflammation in Peripheral joints and Entheses (MRI-WIPE) [1] has been developed and validated for the entire body assessment, including the knee, but not separately validated for the knee joint region. Detailed MRI scoring systems exist for heels, hands and feet, but although knee arthritis is a key cause of functional impairment, no detailed scoring system has been validated for inflammatory arthritides.
The Knee Inflammation MRI Scoring System (KIMRISS) [2] was developed and validated in osteoarthritis and demonstrated good reliability.

Objectives: To perform region-based development of whole-body MRI through validation of two knee region scoring systems in $\mathrm{SpA}$.

Methods: Assessment of inflammation was performed in the knee region on sagittal WB-MRIs using 2 scoring systems, MRI-WIPE and KIMRISS (Figure 1), in 4 iterative multi-reader exercises. In the final exercise, images (psoriatic arthritis, axial and peripheral $\mathrm{SpA}$ ) were obtained before and after TNF-inhibitor.

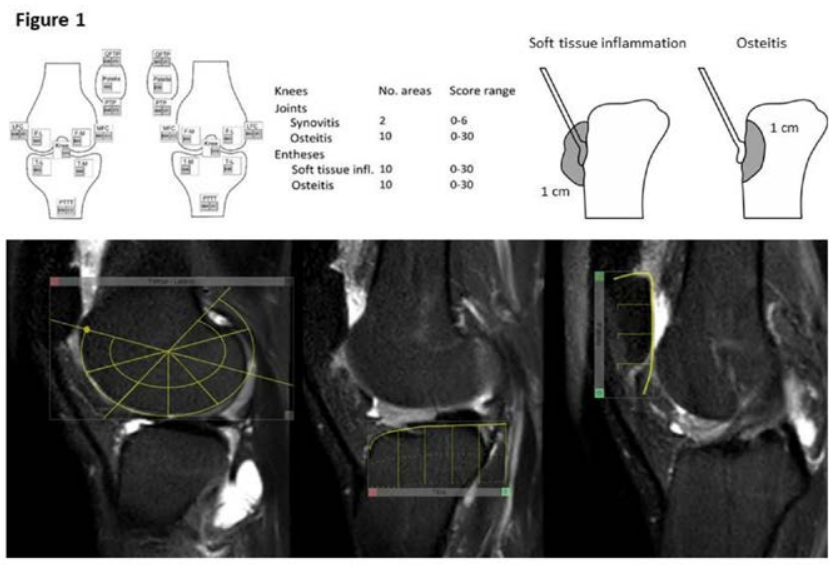

Figure 1. Upper row, left: MRI-WIPE schematic and scoring ranges for the knee-region from Krabbe, et al [1]; Upper row, right: schematic drawing of the principle of scoring osteitis and soft tissue inflammation [1]. Usprated by the tibing

[1]: Upper

sed superimposed interactive overlays used in KIMRISS for osteitis scoring positioned for femur, tibia and patells.

Results: In the final exercise (exercise 4), reliability was mostly good for experienced readers with the overall highest interreader agreement in the previous exercise (exercise 3). Median pairwise single measure intraclass correlation coefficients for osteitis and synovitis/effusion for status/change were $0.71 / 0.48$ (WIPE osteitis), 0.48/0.77 (WIPE synovitis/effusion), 0.59/0.91 (KIMRISS osteitis) and 0.92/0.97 (KIMRISS synovitis/effusion) (Table 1). Wilcoxon signed-rank test showed significant change in synovitis/effusion for both methods and they correlated significantly regarding status in osteitis $(0.92, \mathrm{p}<0.001)$ and synovitis/ effusion $(0.89, p=0.001)$ and change in synovitis/effusion $(0.89, p<0.001)$. Stand ardized response mean was 0.74 (WIPE synovitis/effusion) and 0.78 (KIMRISS synovitis/effusion).

Conclusion: MRI-WIPE and KIMRISS may both be useful as part of modula whole-body evaluation in clinical studies.

REFERENCES:

[1] Krabbe S et al. J Rheum. 2019;46(9):1215-21

[2] Jaremko JL et al. RMD Open. 2017;3(1):e000355

Acknowledgements: We thank CARE Aarthritis Limited (carearthritis.com) for help with setting up the web-based scoring interface, the scoring exercises, and the web-based meetings. We thank all who participated in the SIG (Special Interest Group) virtual OMERACT meeting 29 October 2020. HMO, GDM and PGC are supported in part by the National Institute for Health Research (NIHR) Leeds Biomedical Research Centre, United Kingdom. The views expressed in this study are those of the authors and not necessarily those of the NHS, the NIHR or the Department of Health.

Disclosure of Interests: Marie Wetterslev: None declared, Walter P Maksymowych Speakers bureau: AbbVie, Janssen, Novartis, Pfizer and UCB,

Table 1. MRI-WIPE knee and KIMRISS interreader reliability for OMERACT exercises 3 and 4

\begin{tabular}{|c|c|c|c|c|c|c|c|c|c|c|}
\hline \multirow[b]{3}{*}{ Variables } & \multirow[b]{3}{*}{ No. patients } & \multirow[b]{3}{*}{ Type of score } & \multicolumn{4}{|c|}{ MRI-WIPE Knee } & \multicolumn{4}{|c|}{ KIMRISS } \\
\hline & & & \multicolumn{2}{|c|}{ Osteitis } & \multicolumn{2}{|c|}{ Synovitis/effusion } & \multicolumn{2}{|c|}{ Osteitis } & \multicolumn{2}{|c|}{ Synovitis/effusion } \\
\hline & & & Mean score & ICC & Mean score & ICC & Mean score & ICC & Mean score & ICC \\
\hline Exercise 3 & $\begin{array}{l}11 \\
11\end{array}$ & $\begin{array}{l}\text { Status } \\
\text { Change }\end{array}$ & $\begin{array}{c}3.6(0-16) \\
1.1(-2-6)\end{array}$ & $\begin{array}{c}0.57(-0.06-0.98) \\
0.53(0.03-0.90)\end{array}$ & $\begin{array}{l}1.8(0-4) \\
0(-2-1)\end{array}$ & $\begin{array}{c}0.47(0.05-0.85) \\
0.32(-0.13-0.76)\end{array}$ & $\begin{array}{l}32.3(1-224) \\
27.7(-9-131)\end{array}$ & $\begin{array}{c}0.87(0.66-0.99) \\
0.58(-0.30-0.96)\end{array}$ & $\begin{array}{l}29.9(11-60) \\
-1.6(-33-11)\end{array}$ & $\begin{array}{l}0.34(-0.62-0.87) \\
0.48(-0.32-0.95)\end{array}$ \\
\hline 9 readers & & & & & & & & & & \\
\hline Exercise 3 & $\begin{array}{l}11 \\
11\end{array}$ & $\begin{array}{l}\text { Status } \\
\text { Change }\end{array}$ & $\begin{array}{l}3.1(0-16) \\
0.9(-3-6)\end{array}$ & $\begin{array}{l}0.83(0.71-0.97) \\
0.72(0.57-0.83)\end{array}$ & $\begin{array}{c}2.5(0-5) \\
0(-2-1)\end{array}$ & $\begin{array}{l}0.59(0.51-0.71) \\
0.63(0.49-0.76)\end{array}$ & $\begin{array}{c}34.4(0-233) \\
19.3(-23-86)\end{array}$ & $\begin{array}{l}0.89(0.83-0.99) \\
0.46(0.18-0.83)\end{array}$ & $\begin{array}{l}36.5(16-78) \\
-1.8(-45-17)\end{array}$ & $\begin{array}{l}0.59(0.08-0.86) \\
0.89(0.82-0.95)\end{array}$ \\
\hline 3 readers & & & & & & & & & & \\
\hline Exercise 4 & $\begin{array}{l}10 \\
20\end{array}$ & $\begin{array}{l}\text { Change } \\
\text { Status }\end{array}$ & $\begin{array}{c}-0.25(-4-5) \\
2.9(0-7)\end{array}$ & $\begin{array}{l}0.38(-0.35-0.94) \\
0.50(-0.01-0.84)\end{array}$ & $\begin{array}{c}-1.0(-3-1) \\
2.1(0-4)\end{array}$ & $\begin{array}{l}0.30(-0.43-0.89) \\
0.44(-0.21-0.79)\end{array}$ & $\begin{array}{c}-0.45(-37-65) \\
15.2(0-66)\end{array}$ & $\begin{array}{l}0.26(-0.86-0.97) \\
0.35(-0.04-0.89)\end{array}$ & $\begin{array}{c}-14.7(-48-0.20) \\
55.6(1-122)\end{array}$ & $\begin{array}{c}0.48(-0.39-0.99) \\
0.54(0.01-0.96)\end{array}$ \\
\hline 9 readers & & & & & & & & & & \\
\hline Exercise 4 & $\begin{array}{l}10 \\
20\end{array}$ & $\begin{array}{l}\text { Change } \\
\text { Status }\end{array}$ & $\begin{array}{l}0.2(-2-6) \\
2.3(0-6)\end{array}$ & $\begin{array}{l}0.48(0.16-0.66) \\
0.71(0.60-0.80)\end{array}$ & $\begin{array}{c}-1.4(-5-0) \\
2.7(0-5)\end{array}$ & $\begin{array}{l}0.77(0.70-0.82) \\
0.48(0.42-0.57)\end{array}$ & $\begin{array}{l}5.8(-27-111) \\
11.4(0-36)\end{array}$ & $\begin{array}{l}0.92(0.90-0.94) \\
0.59(0.39-0.71)\end{array}$ & $\begin{array}{c}-20.7(-65-28) \\
69.4(1-153)\end{array}$ & $\begin{array}{l}0.97(0.96-0.98) \\
0.91(0.87-0.93)\end{array}$ \\
\hline
\end{tabular}

